\title{
Comparative Study of Frequency of Micronuclei Using Papanicolaou Stain in Potentially Malignant Disorders and Oral Squamous Cell Carcinoma
}

\author{
R. Shravani, N. Vaishali, P. Preethi, R. Poornima, K. C. Shobhita, M. Priyanka \\ Department of Oral and Maxillofacial Pathology, Government Dental College and Hospital, Hyderabad, Telangana, India \\ Email for correspondence: renukuntla.shravani@gmail.com
}

\begin{abstract}
Background: Early recognition is crucial for successful treatment and good prognosis of oral cancer. A simple, noninvasive, and reasonably effective technique for initial evaluation of a suspicious oral lesion are exfoliative cytology. Micronuclei being structures presenting after genomic damage can be used as a screening tool in early detection of cancer. Method: A total of 60 subjects, 30 with potentially malignant disorders (PMD) (oral lichen planus and oral submucous fibrosis), 15 with oral squamous cell carcinoma (OSCC), and 15 with apparently normal mucosa, were included in the study. Cytological smears were collected from these groups and micronuclei were examined using Papanicolaou stain. One-way ANOVA was used to compare the mean micronuclei distribution among different study groups. Results: An increase in the frequency of micronuclei from normal to PMD to cancerous lesions was observed. Analysis has shown significant differences between various study groups $(P<0.05)$, with highest score observed in OSCC group, intermediary in PMD, and least in controls. Conclusion: The oral mucosal micronuclei can be used as a marker of epithelial carcinogenic progression.
\end{abstract}

Key words: Lichen planus, micronuclei, oral potentially malignant disorders, oral squamous cell carcinoma, Papanicolaou stain

\section{INTRODUCTION}

Cancer is considered as one of the most common life-threatening diseases affecting the mankind. It is estimated that oral cancer is the sixth most common cause of mortality and highest incidence is seen in the Indian population. ${ }^{[1]}$ The term oral cancer is nearly synonymous with oral squamous cell carcinoma (OSCC) because of its great numerical dominance. ${ }^{[2]}$ Most of the oral cancers are preceded by the potentially malignant disorders (PMD) that give a warning of risk and provide an opportunity

\section{Quick Response Code Article Info:}

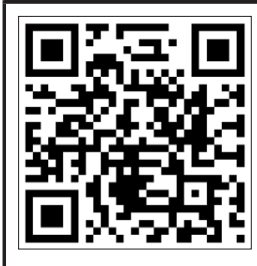

doi: 10.5866/2018.10.10001

Received: 28-12-2017

Revised: 29-01-2018

Accepted: 08-02-2018

Available Online: 15-04-2018, 2017 (www. nacd.in)(C NAD, 2017 - All rights reserved for detection and preventive measures. ${ }^{[3]}$ The possibility of malignant transformation of PMD is reported to be between $6.6 \%$ and $36.4 \% .{ }^{[4]}$ Early diagnosis may improve the survival and decrease the morbidity of patients. ${ }^{[3]}$ Oral cancer is attributed to complex karyotypes that include many chromosomal deletions, translocations, and structural abnormalities. ${ }^{[5]}$

Micronuclei can be used as an indicator of genotoxic exposure as shown in the study carried out by Heddle et al. (1983). ${ }^{[6]}$ Micronucleus can be defined as a microscopically visible, round-to-oval cytoplasmic chromatin mass next to the nucleus. These are extranuclear cytoplasmic bodies formed by the exclusion of chromosome fragments or whole chromosomes lagging at mitosis. Micronuclei being structures presenting after genomic damage in buccal mucosal cells are good indicators of chromosomal alterations in cytological samples. ${ }^{[3]}$ 
The study was undertaken to ascertain the frequency of Micronuclei in oral exfoliated cells of normal individuals, patients with PMDs and OSCC and to assess the role of Micronuclei in diagnosing malignant transformation of PMD.

\section{MATERIALS AND METHODS}

A retrospective study was conducted with a sample size of 60 subjects which included 30 subjects with PMD (oral submucous fibrosis and oral lichen planus), 15 subjects with OSCC, and 15 subjects with normal mucosa.

\section{Inclusion criteria}

Patients with apparently normal mucosa were included in the study.

Clinically and histopathologically diagnosed cases of as follows:

1. Oral submucous fibrosis,

2. Oral lichen planus, and

3. OSCC.

\section{Exclusion Criteria}

1. Patients detected with malignancies other than OSCC.

2. Patients with a history of malignancy and under treatment for malignancy (surgery, chemotherapy, and radiotherapy).

3. Patients with metastatic tumors in jaws from systemic malignancies.

\section{Sample collection}

\section{Collection of exfoliated cells ${ }^{[3]}$}

Subjects were asked to rinse their mouth gently with tap water before the start of the procedure. A slightly moistened wooden spatula was used to obtain the smear of exfoliated cells from the oral cavity (buccal mucosa in control group). Oral mucosal cells for obtaining the smear were scraped from the margins of the lesion. Pre-cleaned microscopic slides were used for smear preparation on which the cells were coated and fixed in alcohol for $15 \mathrm{~min}$.

\section{Cytological preparation and evaluation}

Papanicolaou technique for staining of the smears was used. Screening of each slide was made in a Zigzag manner from one end to the other end of the slide. From each slide, 200 cells were examined using low magnification (10) for screening and high magnification (40) for counting of micronuclei under the light microscope.

\section{Scoring criteria}

The criteria developed by Tolbert et al. which was used for counting the micronuclei, as follows: ${ }^{[7]}$

1. Rounded smooth perimeter suggestive of a membrane.

2. Less than a third the diameter of associated nucleus, but large enough to discern shape and color.

3. Staining intensity similar to nucleus.

4. Same focal plane as nucleus.

5 . Texture similar to nucleus.

6. The absence of overlap with or bridge to the nucleus.

Only those structures fulfilling the abovementioned criteria were recorded as micronuclei.

\section{RESULTS}

An increase in the frequency of micronuclei from normal to PMD to cancerous lesions was observed [Figures 1 and 2].

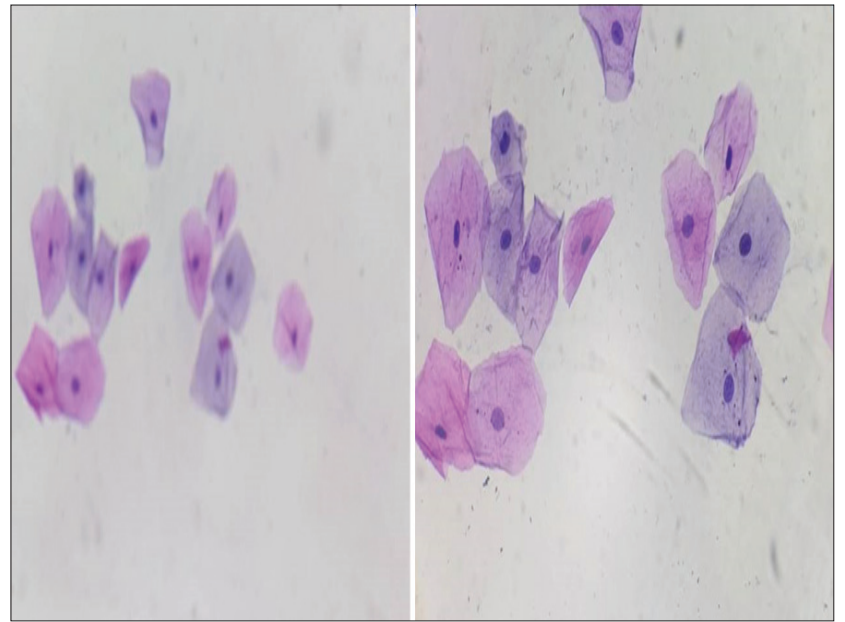

Figure 1: Photomicrograph showing micronuclei in oral potentially malignant disorders by Papanicolaou stain ( 10 and 40$)$

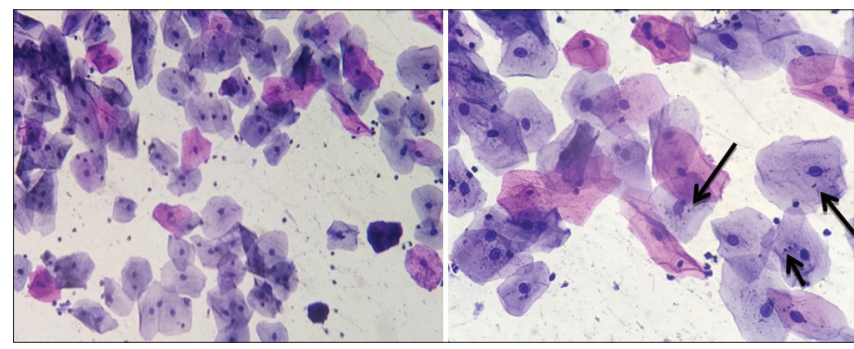

Figure 2: Photomicrograph showing micronuclei in oral squamous cell carcinoma by Papanicolaou stain ( 10 and 40 ) 


\begin{tabular}{lccccc} 
Table 1: Age distribution among the groups & & & \\
Group & $\mathbf{n}$ & Minimum & Maximum & Mean \pm SD & P value \\
\hline Control & 15 & 25.00 & 60.00 & $36.2000 \pm 11.79104$ & P $<0.001$ \\
PMD & 30 & 21.00 & 60.00 & $40.1333 \pm 11.05701$ & \\
OSCC & 15 & 39.00 & 64.00 & $50.4667 \pm 9.65747$ &
\end{tabular}

SD: Standard deviation, PMDs: Potentially malignant disorders, OSCC: Oral squamous cell carcinoma

\section{Table 2: Gender distribution among the groups}

\begin{tabular}{lcc} 
Group & Frequency (\%) & P value \\
\hline Controls & & \\
Male & $6(40.0)$ & \\
Female & $9(60.0)$ & $\mathrm{P}<0.01$ \\
Total & $15(100.0)$ & \\
PMD & & \\
Male & $21(70.0)$ & \\
Female & $9(30.0)$ & \\
Total & $30(100.0)$ & \\
OSCC & & \\
Male & $13(86.7)$ & \\
Female & $2(13.3)$ & \\
Total & $15(100.0)$ &
\end{tabular}

PMDs: Potentially malignant disorders, OSCC: Oral squamous cell carcinoma

One-way ANOVA test was performed among subjects of different age groups and found mean age distribution among controls, PMD, and OSCC was $36.2,40.1$, and 50.4 years respectively, the difference being statistically significant $(P<0.05)$ [Table 1 and Graph 1].

Gender distribution among different groups have shown $40 \%$ males and $60 \%$ females in controls, $70 \%$ males and $30 \%$ females in PMD, and among OSCC group $86.7 \%$ were male and $13.3 \%$ were female. Pearson's Chi-square test was done between gender distribution among these groups, and a statistically significant $(P<0.05)$ correlation was observed [Table 2 and Graph 2].

Mean distribution of micronuclei among controls, PMD, and OSCC was 19.4,32.0, and 56.7, respectively, and a one-way ANOVA was computed to estimate the mean micronuclei distribution among different study groups. This difference was significant statistically $(P<0.05)$ [Table 3 and Graph 3].

The results analyzed with post hoc test between controls and PMD $(P=0.00)$, controls and $\operatorname{OSCC}(P=0.00)$, and PMD and OSCC $(P=0.00)$

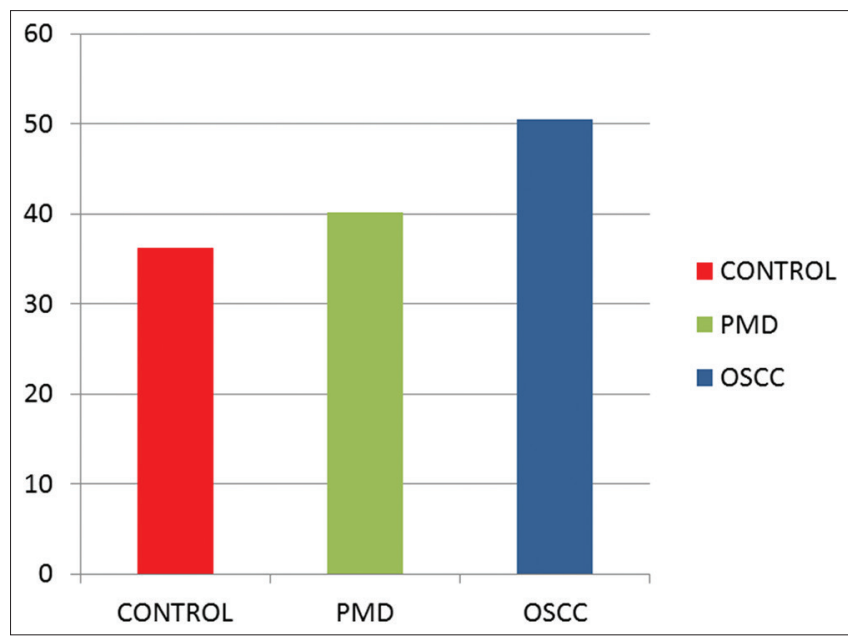

Graph 1: Age distribution among the groups

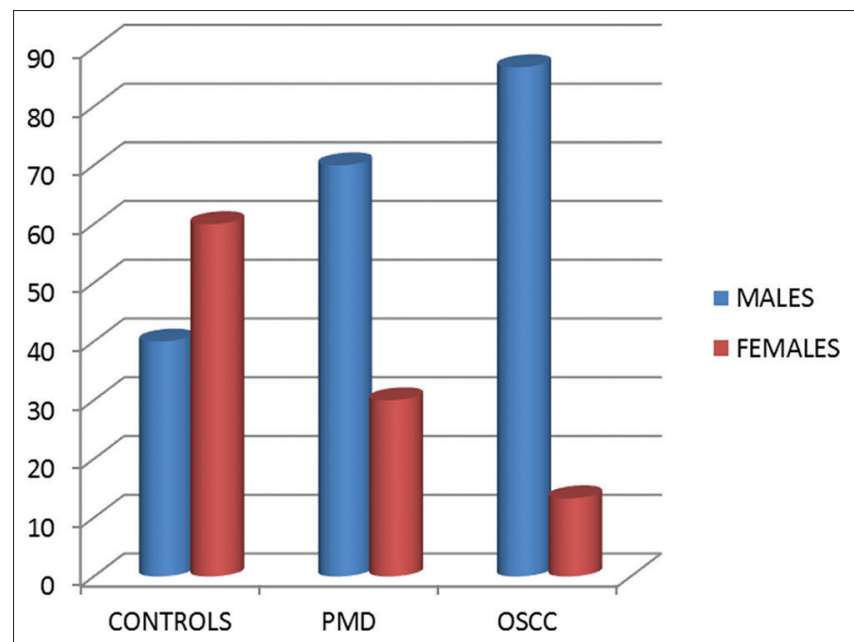

Graph 2: Gender distribution among the groups

were statistically significant and insignificant among potentially malignant groups (0.927) [Table 4].

\section{DISCUSSION}

OSCC is the most common cancer and accounts for $90 \%$ to $95 \%$ of all oral malignancies. Exfoliative cytology is a valuable tool for mass screening purposes, with a sensitivity of $94 \%$, 


\begin{tabular}{|c|c|c|c|c|c|c|c|}
\hline \multirow[t]{2}{*}{ Group } & \multirow[t]{2}{*}{$\mathbf{n}$} & \multirow[t]{2}{*}{ Mean } & \multirow[t]{2}{*}{ Std. deviation } & \multirow[t]{2}{*}{ Std. error } & \multicolumn{2}{|c|}{$95 \%$ confidence interval for mean } & \multirow[t]{2}{*}{ ANOVA } \\
\hline & & & & & Lower Bound & Upper Bound & \\
\hline Controls & 15 & 19.4000 & 3.66060 & 0.94516 & 17.3728 & 21.4272 & $\begin{array}{c}\mathrm{F}=320.535 \\
\mathrm{P}<0.001\end{array}$ \\
\hline PMD & 30 & 32.0000 & 3.53309 & 0.64505 & 30.6807 & 33.3193 & \\
\hline OSCC & 15 & 56.7333 & 5.53517 & 1.42917 & 53.6681 & 59.7986 & \\
\hline
\end{tabular}

PMDs: Potentially malignant disorders, OSCC: Oral squamous cell carcinoma

Table 4: Mean difference of micronuclei among different groups

Dependent Variable: Micronuclei (Tukey HSD)

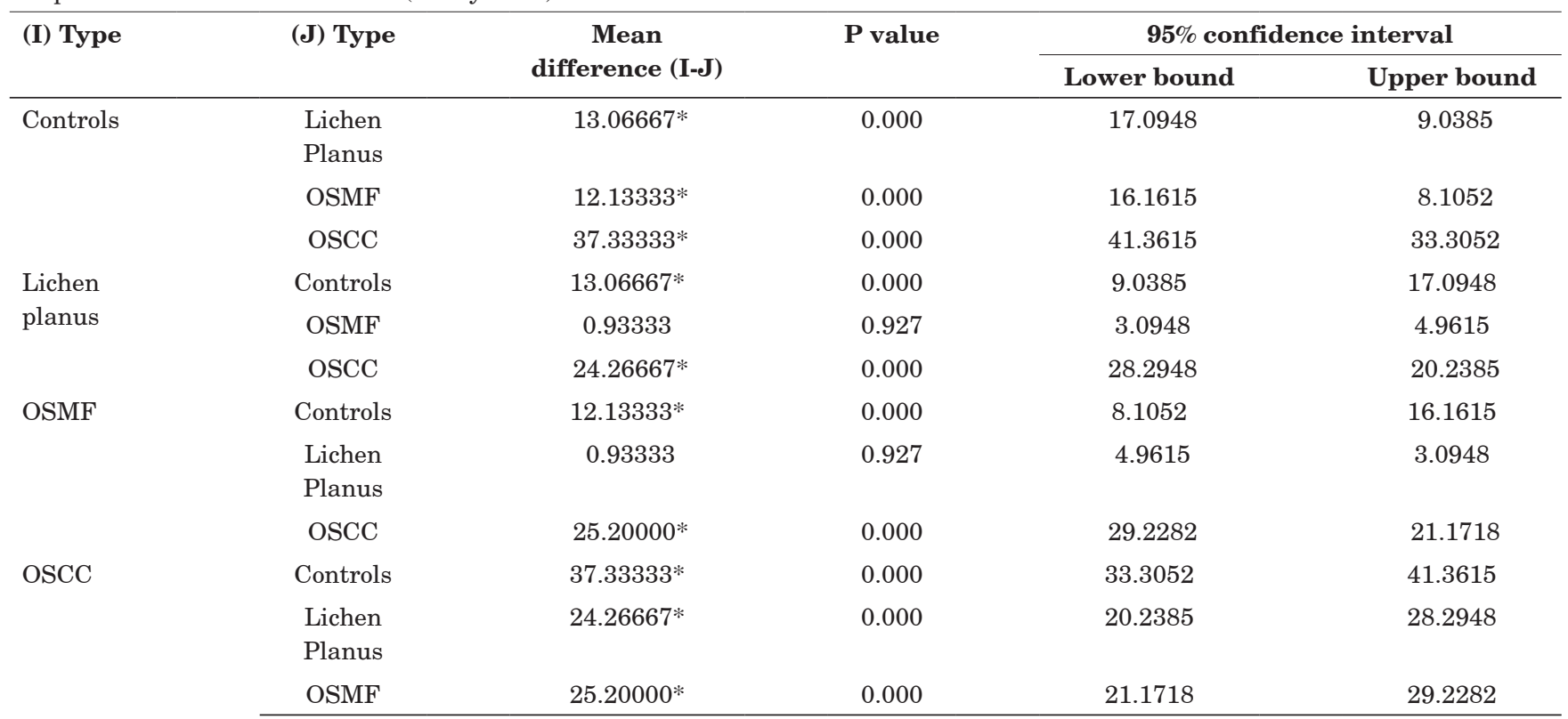

*The mean difference is significant at the 0.05 level. OSMF: Oral leukoplakia, submucous fibrosis, OSCC: Oral squamous cell carcinoma

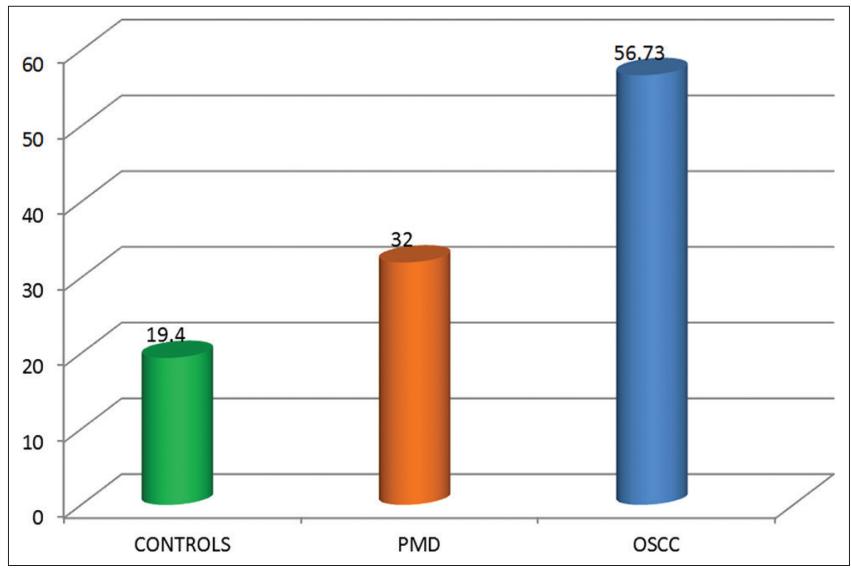

Graph 3: Distribution of micronuclei among the groups

specificity of $100 \%$, and an accuracy of $100 \%$ in detection of OSCC. ${ }^{[8,9]}$ A variety of substances, including genotoxic agents and carcinogenic compounds in tobacco, betel nut, and alcohol, induce the formation of micronuclei in oral exfoliated cells. ${ }^{[10]}$ Micronuclei formation can occur by two basic phenomena in mitotic cells, which are chromosome breakage and dysfunction of the mitotic apparatus. They can be formed from eccentric chromosomes, chromatid fragments, whole chromosomes, or chromatids that lag behind in anaphase and are left outside the daughter nuclei in telophase. ${ }^{[11]}$

The difference in the mean percentage of Micronuclei in PMD and controls was found to be statistically significant. Similar results were noticed in Sangle et al. ${ }^{[3]}$ The possible illustration for Micronuclei formation in preneoplastic conditions includes chromosome loss or breakage, chromosomal aberrations, mitotic apparatus dysfunctions, aneuploidy, and genetic instability. 
A statistically significant $(\mathrm{P}<0.05)$ increase in the occurrence of micronuclei from control to PMD to OSCC group was observed. These observations indicate an increase in cytogenetic damage of the epithelial cells. This is in accordance with the studies conducted by Sangle et al., Devi et al., and Halder et al. ${ }^{[3,12,13]}$ The mean difference between the numbers of Micronuclei in PMD group and OSCC group was statistically highly significant $(\mathrm{P}<0.05)$. This is similar to the study conducted by Gupta et al. (2011). ${ }^{[14]}$

The study conducted by Casartelli et al. concluded that the gradual increase in micronuclei frequency from normal mucosal to pre-cancerous lesion to carcinoma proposed a link of this biomarker with neoplastic progression. ${ }^{[15]}$ There was stepwise increase found in the percentage of micronucleated cells and micronuclei from control to pre-cancer patients and from pre-cancer to cancer patients in the studies by Saran et al and Grover et al. ${ }^{[16-17]}$

In the study conducted by Kumar et al., the cells from OSCC lesions were obtained by mincing the biopsy tissue and preparing single cell suspensions from it. ${ }^{[10]}$ This is in contrast to the present study where the smears were made by exfoliative cytology.

A statistical insignificance $(P=0.92)$ was seen among the PMD groups (lichen planus and OSMF). This is attributed to the fact that the rates of chromosomal aberrations in both these lesions were almost similar as stated by the Sarode et al. (2004).

\section{Limitations}

1. Smaller sample size.

2. Staining and counting of micronuclei is a technique sensitive procedure.

3 . We used only single observer.

4. Inclusion of different grades of OSCC might give an accurate result.

\section{Future prospects}

1. Estimation of micronuclei with various nuclear stains.

2. Relative assessment of micronuclei with various morphometric parameters

\section{CONCLUSION}

With the findings of the current study, it is apparent that there is a gradual increase in the frequency of micronuclei from normal to precancerous to cancerous lesions. Although the sample size was small, it can be concluded that oral mucosal micronuclei may be used as a marker for epithelial carcinogenic progression.

\section{REFERENCES}

1. Hande A, Mimansha P, Aakruti A, Minal C, Suchitra G, Swati P. Diagnostic Accuracy of the acridine orange fluorescence staining and papanicolaou staining in detection of oral cancer. J Int Acad Res Multidiscip 2015;3:245-55.

2. Prakash N, Sharada P, Pradeep GL, Soundarya N. Reliability of acridine orange fluorescence microscopy in oral cytodiagnosis. Indian J Dent Res 2011;22:649-53.

3. Sangle VA, Bijjaragi S, Shah N. Comparative study of frequency of micronuclei in normal, potentially malignant diseases and oral squamous cell carcinoma. J Nat Sci Bio Med 2016;7:33-8.

4. Shashikala R, Indira AP, Manjunath GS. Role of micronucleus in exfoliative cytology. J Pharm Bioallied Sci 2015;7:409-13.

5. Palaskar S, Jindal C. Evaluation of Micronuclei using papanicolaou and May Grunwald-Giemsa stain in individuals with different tobacco habits: A comparative study. J Clin Diagn Res 2011;4:3607-13.

6. Heddle JA, Hite M, Kirkhart B, Mavournin K, MacGregor JT, Newell GW, et al. The induction of micronuclei as a measure of genotoxicity. A report of the U.S. Environmental protection agency genetox program. Mutat Res1983;123:61-118.

7. Tolbert PE, Shy CM, Allen JW. Micronuclei and other nuclear anomalies in buccal smears: A field test in snuff users. Am J Epidemiol 1991;134:840-50.

8. Palve DH, Tupkari JV. Clinicopathological correlation of micronucleus in oral squamous cell carcinoma by exfoliative cytology. J Oral Maxillofac Pathol 2008;12:2-7.

9. Ramaesh T, Ratnatunga N, Mendis BR, Rajapksa S. Exfoliative cytology in screening for malignant and precancerous lesions in the buccal mucosa. Ceylon Med J 1998;43:206-9.

10. Kumar V, Rao NN, Nair NS. Micronuclei in oral squamous cell carcinoma: A marker of genotoxic damage. Indian $\mathrm{J}$ Dent Res 2000;11:101-6.

11. Jadhav K, Gupta N, Ahmed MB. Micronuclei: An essential biomarker in oral exfoliated cells for grading of oral squamous cell carcinoma. J Cytol 2011;28:7-12.

12. Devi P, Thimmarasa VB, Mehrotra V, Arora P. Micronucleus assay for evaluation of genotoxicity in potentially malignant and malignant disorders. J Ind Acad Oral Med Rad 2011;23:97-100.

13. Halder A, Chakraborty T, Mandal K, Gure PK, Das S, Raychowdhury R, et al. Comparative study of exfoliated oral mucosal cell micronuclei frequency in normal, precancerous and malignant epithelium. Int J Hum Genet 2004;4:257-60

14. Gupta A, Bhovi TV, Jaju P. Micronuclei frequency as an early diagnostic tool for detection of oral cancer: A comparative study. Intl J Oral Health Dent 2016;2:77-83. 
15. Casartelli G, Bonatti S, De Ferrari M, Scala M, Mereu P, Margarino $\mathrm{G}$, et al. Micronucleus frequencies in exfoliated buccal cells in normal mucosa, precancerous lesions and squamous cell carcinoma. Anal Quant Cytol Histol 2000;22:486-92.

16. Saran R, Tiwari RK, Reddy PP, Ahuja YR. Risk assessment of oral cancer in patients with pre-cancerous states of the oral cavity using micronucleus test and challenge assay. Oral Oncol 2008;44:354-60.

17. Grover S, Mujib AB, Jahagirdar A. A comparative study for selectivity of micronuclei in oral exfoliated epithelial cells. J Cytol 2012;29:230-5. 